\title{
Relación entre el rendimiento y la ansiedad en el deporte: una revisión sistemática Relationship between performance and anxiety in sports: a systematic review
}

\author{
Antonio Núñez Prats, Alejandro Garcia Mas \\ Universidad de las Islas Baleares (España)
}

\begin{abstract}
Resumen. El rendimiento deportivo y su relación con la ansiedad competitiva es un tema recurrente en los estudios de psicología del deporte. La idea de que la ansiedad influye de alguna manera sobre el rendimiento de los deportistas, normalmente de forma negativa, ha estado siempre presente a pesar de no concretarse de qué manera afecta esta al rendimiento y de no contar con el apoyo empírico necesario. En el presente artículo se realiza una revisión sistemática usando la base de datos Web of Knowledge, con el objetivo de clarificar el estado actual de la idea, cuales son los marcos teóricos predominantes y que dicen los resultados empíricos al respecto. Se realiza un recorrido por las etapas llevadas a cabo desde la selección de las palabras clave, la selección de la base de datos, pasando por la criba de los artículos y los criterios de inclusión, hasta llegar a los resultados finales, lo cuales indican que no existen evidencias empíricas y/o experimentales suficientes para esclarecer la relación entre ansiedad y rendimiento deportivo.

Palabras clave: rendimiento, ansiedad, criterio, estrés y activación.
\end{abstract}

Abstract. Athletic performance and their relationship with competitive anxiety is a recurring theme in sport psychology studies. The idea that somehow anxiety influences on the performance of athletes has always been present despite not understand how this affects performance, and despite not having the empirical support. In this article a systematic review is performed using the database Web of Knowledge, in order to clarify the current status of the idea, which are the dominant theoretical frameworks and empirical say about results. A journey is made by the steps performed from the selection of keywords, selecting the database, through the sieve of the articles and the criteria for inclusion, reaching the final results, which indicate that there is insufficient evidence to clarify the relationship between sports performance and anxiety

Keywords: performance, anxiety, criteria, stress and arousal.

\section{Introducción}

El concepto de rendimiento deportivo está estrechamente vinculado al deporte de competición, ya que este último, exige que los deportistas exploten sus recursos al máximo. Cuando nos referimos al deporte de competición estamos aludiendo al deporte de alto rendimiento, $\mathrm{y}$ no al deporte de iniciación en etapas tempranas de la vida de los deportistas.

De entre las muchas definiciones existentes, podríamos entender al rendimiento deportivo como el resultado de una actividad deportiva, dentro del deporte de competición según unas reglas previamente establecidas (Martin, 2001).

Este concepto de rendimiento deportivo debe entenderse de forma global, ya que engloba como antecedentes factores como la preparación física, la preparación táctica, la preparación técnica y la preparación psicológica (Buceta, 1998).

En los inicios de la psicología científica como tal, los estudios experimentales de W. Wundt (Wundt, 1874) se dirigían ya a estudiar algunas de las variables psicofisiológicas, que posteriormente se podían relacionar con el rendimiento psicofísico humano.

En dichos estudios Wundt estudiaba, de forma objetiva, los procesos perceptivos y los tiempos de reacción de los sujetos. Se demostró que la latencia entre estímulo y respuesta se halla sujeta a la influencia de diversos factores como son la atención, el aprendizaje, la fatiga, el consumo de drogas o medicamentos, la edad del sujeto, el incentivo, castigos específicos, etc. (Wundt, 1874)

Estos pioneros estudios han servido de pilar indiscutible para asentar las bases y favorecer el crecimiento de la psicología como ciencia, y concretamente, para la comprensión de cómo el sujeto percibe y experimenta los estímulos que le rodean.

Estos primeros estudios de Wundt, sirvieron de antesala para posteriores trabajos, como los de Fechner (Fechner, 1966), que trataban de explicar por medio del desarrollo de una formulación matemática, la relación entre la sensación y la percepción del estímulo con la intensidad de este, lo que se ha conocido con el paso del tiempo como la «Ley de Weber-Fechner».

Desde una perspectiva psicológica existen evidencias intuitivas sobre la relación existente entre los estados psicológicos y el rendimien-

Fecha recepción: 11-05-16. Fecha de aceptación: 11-02-17

Antonio Núñez Prats

toni.nunezprats@gmail.com to deportivo (Sánchez, González, Ruiz, San Juan, Abando, Nicolás \& García, 2001). Las variables psicológicas que influyen en el rendimiento deportivo, como son los estados de ánimo, las expectativas, el nivel de activación y la ansiedad en situación de competición, han sido objeto de numerosos y exhaustivos estudios desde la Psicología del deporte (LeUnes, Hayward \&

Davis, 1988).

Desde una perspectiva psico-fisiológica, es de sobras conocido el papel que desempeña el nivel de activación o arousal en el desarrollo y preparación de tareas psicomotrices.

La activación o arousal es el estado energético del organismo que facilita las funciones de atención, emoción y los procesos cognitivos. En general se considera un estado de disposición para la acción.

La activación puede ser relativamente global situándose en un continuo desde el estado de somnolencia a la máxima alerta. La activación pone en marcha, de manera integrada el sistema nervioso en su conjunto, tanto el central como el sistema nervioso autónomo (SNA). Dentro del SNA, la rama simpática está orientada a facilitar la acción motora, mientras que la rama parasimpática se dirige preferentemente a funciones vegetativas actuando para restringir el arousal a su nivel homeostático (Pozo, Cortes \& Martín, 2013).

La competición en sí misma, es una fuente poderosa de activación psicofísica, y, eventualmente de estrés. El rendimiento que manifiesta el deportista durante los entrenamientos no se ve sometido a los mismos condicionantes que el rendimiento manifestado durante la competición.

Del rendimiento manifestado durante la competición va a depender el éxito profesional, la autoestima, la valoración social, la economía del club al que pertenece e incluso el prestigio de su comunidad o país (Gutiérrez, Estévez, García \& Pérez, 1997), por tanto, el nivel competitivo de los deportistas puede condicionar el grado de ansiedad experimentada (Ruiz-Juan, Zarauz \& Flores-Allende, 2016).

Es por ello que la competición supone un desafío, una amenaza, en la que se manifiestan reacciones prototípicas de ansiedad, con sus respectivas consecuencias para el rendimiento, y la competición.

Las concepciones clásicas de ansiedad competitiva, la concebían, como una reacción emocional negativa, fruto de la discrepancia entre la demanda del ambiente y la capacidad de respuesta del sujeto, y diferenciaban entre ansiedad estado y ansiedad rasgo, aludiendo al carácter transitorio o estable de la misma (Martens, 1977). Estas concepciones han evolucionado hacia modelos más complejos (Smith \& Smoll, 2004) en el que la ansiedad es entendida en base a tres componentes; ansiedad somática, ansiedad cognitiva y pérdida de concentración. En la adaptación a población española del SAS-2 (Ramis, Torregrosa, Vilarich \& 
Cruz, 2010), los tres componentes propuestos por Smith y Smoll, pasaron a denominarse; ansiedad somática, preocupación y desconcentración.

Es dentro de este último marco conceptual donde podemos situar el reciente estudio con nadadores que sirve como ejemplo, coincidiendo con la literatura clásica, de cómo la ansiedad competitiva tiene un efecto bloqueador o debilitante del rendimiento deportivo. La respuesta ansiógena está formada por tres componentes, que se conoce también como el triple canal de respuesta; motora, fisiológica y cognitiva, poniéndose de relieve en dicho estudio, como el componente más importante, es el factor cognitivo, asociado a la preocupación por el rendimiento (Ponseti, Sesé \& García-Mas, 2016). En otro estudio, estos autores, ponen de relieve las diferencias de género en cuanto al concepto de ansiedad competitiva, poniendo de manifiesto, y en consonancia con la literatura clásica, que las mujeres presentan niveles más elevados de ansiedad competitiva tanto a nivel cognitivo como somático (Ponseti, García-Mas, Cantallops \& Vidal, 2017).

A pesar de esta larga trayectoria, las relaciones entre ansiedad y rendimiento deportivo no quedan claras, hecho que ha llevado a plantear cuál es el estado actual de la cuestión.

En un estudio muy precoz, ya en 1908, Yerkes y Dodson, establecieron, lo que hoy en día se conoce como la Ley de Yerkes-Dodson, que relaciona el nivel de activación o arousal, con el rendimiento en una tarea dada.

Según esta ley, la relación entre activación y rendimiento tiene forma de U invertida, lo cual significa que existe un punto óptimo de activación para la obtención del máximo rendimiento, y que por encima o por debajo de dicho punto el rendimiento decrece, sea por excesiva activación o por falta de activación, pero que dicho estado o punto óptimo depende primariamente de la relación tarea-sujeto a distintos niveles, como se desprende del concepto de entropía y de las teoría de los sistemas dinámicos.

Esta «ley» fue duramente criticada por el concepto tan general y vago de arousal, y surgieron por ello numerosas revisiones más específicas.

En este contexto se propuso que el rango óptimo de ansiedad de la supuesta ley de la U-invertida, estaba en función de las características de la tarea deportiva (Oxendine, 1970). Esta revisión de la teoría propuso que para tareas de motricidad fina y precisión el nivel óptimo de activación sería más bajo, que el nivel óptimo de activación para tareas que requieran un esfuerzo físico considerable.

Más tarde, Sonstroem \& Bernardo (1982), propusieron una variante más individualizada de la teoría de la U-Invertida, afirmando que el nivel medio de ansiedad experimentado por un atleta a lo largo de una serie de competiciones representa su nivel óptimo de ansiedad competitiva.

Por otro lado, otras teorías plantean que es posible que siempre haya un mínimo nivel de ansiedad en tareas psicomotoras de rendimiento y/o competición, puesto que dichas tareas conllevan cierto grado de incertidumbre.

Hanin (1978) propuso lo que él denominaría la «Teoría de la Zona de funcionamiento Óptimo», conocida también como «Zona Individual de Funcionamiento Óptimo» (ZOF o IZOF). Dicha teoría propone que el rendimiento se ve optimizado cuando la ansiedad se sitúa en un rango estrecho o zona óptima. Pero por contraposición a la teoría de la U-invertida, la IZOF propone que dicha ansiedad puede residir en cualquier lugar dentro del continuo de la ansiedad, dependiendo del sujeto, lo cual viene a decir que no existe una zona óptima normativa en cuanto al nivel óptimo de ansiedad necesario para tal o cual deporte, sino que cada individuo tiene una zona óptima de rendimiento.

Ante tal cantidad y variedad de marcos conceptuales y aplicados en relación a la ansiedad y su influencia sobre el rendimiento se hace necesario aunarlos para tener una visión general del estado de dicha cuestión, y una de las metodologías posibles para alcanzar dicho objetivo, es la revisión sistemática.

El concepto de revisión se ha usado de forma muy laxa en los últimos tiempos, es decir, se han hecho revisiones sin una metodología específica contraponiéndose al meta-análisis y su análisis estadístico pormenorizado.

Es en este contexto donde nace el concepto de revisión sistemática, tratando de asentar las bases para que la revisión siga una serie de normas y pautas que aseguren su validez y fiabilidad, al igual que lo hacemos con el resto de las investigaciones.

La revisión sistemática nace ante el espectacular crecimiento de la producción científica y permite conocer qué es lo que se sabe, cuáles son los datos, cuáles son las posiciones teóricas dominantes y cuáles son las conclusiones provisionales sobre una cuestión dada (Botella \& Gambara, 2012).

Desde un punto de vista formal la revisión sistemática intenta aunar y sintetizar los resultados obtenidos de estudios primarios por medio de la aplicación de una serie de estrategias con el fin de limitar los sesgos y los errores:

1. Búsqueda sistemática y exhaustiva de todos los artículos potencialmente relevantes.

2. Selección mediante criterios explícitos y replicables, de los artículos que serán incluidos finalmente en la revisión.

3. Descripción del diseño y ejecución de los estudios originales, la síntesis de los datos obtenidos y la interpretación de los resultados.

Cuando los diferentes trabajos objeto de análisis, no se combinan estadísticamente, reciben el nombre de revisiones sistemáticas, por el contrario, cuando dichos datos se comparan por medio de un proceso estadístico obteniendo así un índice cuantitativo de la magnitud del efecto que cada estudio está investigando, que posteriormente serán integrados, reciben el nombre de meta-análisis.

Es, por tanto, por tales motivos que se ha decidido llevar a cabo una revisión sistemática del State of the Art actual, de las evidencias existentes que ponen en relación la variable ansiedad y la variable rendimiento deportivo, ya que nos permite hacernos una idea del estado actual de la idea científicamente apoyada.

\section{Material y método}

\section{Procedimiento}

Para la presente investigación, se llevó a cabo un proceso de revisión sistemática, ya que la gran dispersión de metodologías y de instrumentos de recolección de datos utilizado, no hace posible el uso eficaz del meta-análisis para responder a nuestra pregunta (Glass, 1976).

Después de analizar las opciones de las que se disponía dónde realizar la búsqueda, se tomó la decisión de realizar la búsqueda de los trabajos en el portal Web of Knowledge (https://webofknowledge.com). Nos referiremos de ahora en adelante, a dicho portal como Web of Science, puesto que Thomson Reuters decidió en 2014 cambiar el nombre por este último (WoS, de ahora en adelante).

Este, es un servicio en línea de información científica, una base de datos multidisciplinar de referencias bibliográficas que permite acceder a las colecciones electrónicas de Thomson Reuters lo que permite una búsqueda global en todas sus bases de datos.

Decidimos seleccionar dicho portal por delante de SCOPUS (https:/ /www.scopus.com), ya que este último posee un catálogo parcial e incompleto de las revistas de alta calidad, por su dependencia de la editorial Elsevier.

También se barajó la posibilidad de usar la española Journal Scholar Metrics (www.journal-scholar-metrics.infoec3.es) desarrollada por el grupo de investigación EC3 de la Universidad de Granada, pero cuenta con un volumen mucho menor de artículos indexados.

Por lo tanto, WoS, nos pareció la base de datos que nos permitía llegar a la mayor cantidad de artículos para así poder obtener una visión del conjunto a nivel mundial del estado de la cuestión.

La selección de las palabras clave, se realizó mediante la creación de un focus group, en el cual, se analizaron las propuestas iniciales, con el objetivo de relacionar el concepto de rendimiento deportivo con el de ansiedad.

Se determinó que el constructo principal de la presente investigación sería el concepto de performance, y que se iba a emparejar con los 
términos, criteria, anxiety, stress y physiology. Se decidió la combinación Performance, criteria, anxiety ya que por un lado contaba con un volumen considerable de trabajos (848), cubría a priori las necesidades del trabajo, y además era la opción en la que aparecían, también, trabajos en español.

Se matizó desligar el concepto de rendimiento del adjetivo «deportivo» puesto que, en otras áreas, como la música, el rendimiento también va ligado al desempeño psicomotor. Además, se sugirió incluir el término «criterio», aludiendo a los criterios de rendimiento que se tienen en consideración en los trabajos, puesto que los criterios de rendimiento como tales, difieren en cada deporte y/o actividad, y para cada investigador.

Hay que destacar también, que fueron excluidos del estudio, aquellos trabajos en los que el rendimiento era puramente académico, sin componentes psicomotores.

Los idiomas en los que se buscaron los artículos fueron inglés y español, teniendo en cuenta que las palabras clave estaban escritas en inglés, a pesar de seleccionar el idioma español en el buscador. Decidimos acorde a nuestro criterio de selección que existieran artículos a priori en las dos lenguas.

El periodo temporal de búsqueda se inició con los trabajos desde 1908, coincidiendo con el año de publicación de la Ley de YerkesDodson hasta diciembre de 2015 incluido, momento que se dio por finalizada la recogida de datos para este trabajo y se inició su análisis.

La búsqueda y selección de los artículos, por tanto, se llevó a cabo por cinco fases bien diferenciadas que se resumen en la Tabla 1 .

\section{Resultados}

\section{Resultados (1 ${ }^{a}$ Fase)}

En la Tabla 2 se puede observar que se realizó un rastreo sucesivo con diferentes emparejamientos de las palabras clave seleccionadas previamente, para sondear el volumen de trabajos que existían en cada emparejamiento.

En la Tabla 3 se observa que la búsqueda de trabajos en español dio como resultado una considerablemente menor cantidad de trabajos que en lengua inglesa. Además, se observa como algunas de las combinaciones no obtuvieron ningún resultado.
Tabla 1.

Cuadro resumen de las fases llevadas a cabo para la selección de los artículos para la revisión sistemática.

Fases

1.Focus group para la selección de las palabras clave

2- Muestreo inicial (tablas 1 y 2)

.Selección de los criterios de búsqueda

1.Selección en base al título

1.Lectura del resumen de los artículos

Lectura en profundidad de los artículos restantes

Tabla

Cuadro resumen del volumen de artículos en inglés, en función de las palabras clave seleccionadas.

Palabras clave

Performance
Performance, criteria

Performance, criteria

Performance, anxiety
Performance, stress

Performance, criteria, anxiety

Performance, criteria, stress

Performance, criteria, anxiety, physiology

Performance, criteria, stress, physiology

Tabla 3.

Cuadro resumen del volumen de artículos en español, en función de las palabras clave seleccionadas.

Palabras clave

Performance

Performance, criteria

Performance, anxiety

Performance, stress

Performance, criteria, anxie

Performance, criteria, stres

Performance, criteria, anxiety, physiology

Performance, criteria, stress, physiology

\section{Resultados (2 ${ }^{a}$ fase)}

En la Tabla 4 se puede observar un cuadro resumen de los 10 artículos finales que pasaron a formar parte de la tercera fase del estudio. En dicho cuadro aparece el nombre del trabajo, el autor o autores del mismo, la fecha de publicación, el área de trabajo (deporte o música), la metodología empleada y las principales conclusiones de cada artículo.

\section{Resultados ( $3^{a}$ fase $)$}

En la Tabla 5 se puede observar como la mayor parte de estudios (50\%) provienen de Estados Unidos, el 20\% de Inglaterra y solo dos de ellos provienen de España (20\%). Como se observa la mayoría de trabajos provienen de centros de estudios e investigaciones anglosajonas.

Tabla 4

Cuadro resumen descriptivo de los trabajos resultantes ordenados por fecha de publicación.

\begin{tabular}{|c|c|c|c|c|c|}
\hline en & Autores & Fecha & $\begin{array}{l}\text { Campo de } \\
\text { estudio }\end{array}$ & Metodología & Conclusiones de los estudios \\
\hline $\begin{array}{l}\text { The assessment and Treatment of Performance Anxiety } \\
\text { in Musicians }\end{array}$ & $\begin{array}{l}\text { Duncan, B. \& } \\
\text { Agras, S. }\end{array}$ & 1991 & Música & $\begin{array}{l}\text { Estudio con tipos de tratamientos para la ansiedad } \\
\text { por el rendimiento en músicos }\end{array}$ & $\begin{array}{l}\text { Estrategias cognitivas> Estrategias conductuales } \\
\text { Buspirona? No mejoras significativas }\end{array}$ \\
\hline $\begin{array}{l}\text { Anxiety and performance in track and field athletes: A } \\
\text { comparison of the inverted-U Hypothesis with Zone of } \\
\text { Optimal Function Theory }\end{array}$ & $\begin{array}{l}\text { Raglin, J. \& } \\
\text { Turner, P. }\end{array}$ & 1992 & Deporte & $\begin{array}{l}\text { Estudio descriptivo, comparando modelos } \\
\text { teóricos relacionando ansiedad y rendimiento }\end{array}$ & $\begin{array}{l}\text { Los hallazgos dan soporte a la teoría ZOF por } \\
\text { delante de la teoría de la U-invertida. }\end{array}$ \\
\hline $\begin{array}{l}\text { The effects of a cognitive Intervention Strategy on } \\
\text { Competitive State Anxiety and Performance in } \\
\text { Semiprofessional Soccer Players }\end{array}$ & $\begin{array}{l}\text { Maynard, I. \& } \\
\text { Smith, M. }\end{array}$ & 1995 & Deporte & $\begin{array}{l}\text { Estudio con grupo de tratamiento (PTC) y grupo } \\
\text { control }\end{array}$ & $\begin{array}{l}\text { PTC, es una técnica efectiva para el manejo de la } \\
\text { ansiedad. } \\
\text { No significativa la relación entre ansiedad y } \\
\text { rendimiento. } \\
\text { Ansiedad somática relacionada con habilidades } \\
\text { físicas, y la ansiedad cognitiva relacionada con el } \\
\text { proceso de toma de decisiones. }\end{array}$ \\
\hline $\begin{array}{l}\text { Variability in precompetition anxiety and performance } \\
\text { in college track and field athletes }\end{array}$ & $\begin{array}{l}\text { Turner, P. \& } \\
\text { Raglin, J. }\end{array}$ & 1996 & Deporte & $\begin{array}{l}\text { Estudio descriptivo, comparando modelos } \\
\text { teóricos que relacionan ansiedad y rendimiento }\end{array}$ & $\begin{array}{l}\text { Indican que la ZOF, es más eficaz que las variantes } \\
\text { de la teoría de la U-invertida examinadas. }\end{array}$ \\
\hline $\begin{array}{l}\text { Anxiety-Performance relationships in cricketers: } \\
\text { testing the zone of optimal functioning hypothesis }\end{array}$ & $\begin{array}{l}\text { Thelwell, R. \& } \\
\text { Maynard, I. }\end{array}$ & 1998 & Deporte & $\begin{array}{l}\text { Estudio descriptivo comparando ansiedad y } \\
\text { rendimiento en jugadores de cricket }\end{array}$ & $\begin{array}{l}\text { Puntuaciones por encima y por debajo de la ZOF, } \\
\text { obtienen mejores resultados en rendimiento. } \\
\text { El presente estudio no da soporte a la ZOF. }\end{array}$ \\
\hline $\begin{array}{l}\text { Psychological treatment of musical performance } \\
\text { anxiety: current status and future directions }\end{array}$ & $\begin{array}{l}\text { McGinnis, A. \& } \\
\text { Milling, L. }\end{array}$ & 2005 & Música & $\begin{array}{l}\text { Estudio de revisión sobre los tratamientos de la } \\
\text { ansiedad por el rendimiento en músicos }\end{array}$ & $\begin{array}{l}\text { Los tratamientos de exposición y de restructuración } \\
\text { cognitiva son los de elección. Poca literatura al } \\
\text { respecto. }\end{array}$ \\
\hline $\begin{array}{l}\text { Sport-related performance anxiety in Young female } \\
\text { atheletes }\end{array}$ & $\begin{array}{l}\text { Patel, D., Omar, } \\
\text { H. \& Terry, M. }\end{array}$ & 2010 & Deporte & $\begin{array}{l}\text { Estudio de revisión sobre la definición, } \\
\text { epidemiología, evaluación y manejo de la } \\
\text { ansiedad en deportistas femeninas jóvenes }\end{array}$ & $\begin{array}{l}\text { La ansiedad por el rendimiento es común en atletas } \\
\text { femeninas y jóvenes. } \\
\text { El tratamiento multimodal que integra la } \\
\text { psicoterapia es la elección principal } \\
\text { Si los medicamentos fueran necesarios los ISRS } \\
\text { son el tratamiento de elección }\end{array}$ \\
\hline $\begin{array}{l}\text { Free improvisation and performance anxiety among } \\
\text { piano students }\end{array}$ & Allen, R. & 2011 & Música & $\begin{array}{l}\text { Estudio descriptivo de la ansiedad por el } \\
\text { rendimiento ante la improvisación }\end{array}$ & $\begin{array}{l}\text { La “libertad de expresión" reduce la ansiedad por } \\
\text { el rendimiento. } \\
\text { Propone la improvisación como tratamiento para la } \\
\text { ansiedad por el rendimiento. }\end{array}$ \\
\hline $\begin{array}{l}\text { Music performance anxiety-Part } 1 . \text { A review of its } \\
\text { epidemiology }\end{array}$ & Ortíz, A. & 2011 & Música & $\begin{array}{l}\text { Estudio de revisión sobre la epidemiología de la } \\
\text { ansiedad por el rendimiento en músicos de todas } \\
\text { las edades y niveles }\end{array}$ & $\begin{array}{l}\text { El rendimiento musical en niños genera más } \\
\text { ansiedad que el rendimiento en otras tareas } \\
\text { (deporte, tareas cognitivas etc.) } \\
\text { En general los músicos experimentan de forma } \\
\text { frecuente ansiedad por el rendimiento. }\end{array}$ \\
\hline $\begin{array}{l}\text { Music performance anxiety- Part 2: A review of } \\
\text { treatment options }\end{array}$ & Ortíz, A. & 2011 & Música & $\begin{array}{l}\text { Estudio de revisión sobre las posibilidades de } \\
\text { tratamiento de la ansiedad por el rendimiento en } \\
\text { músicos }\end{array}$ & $\begin{array}{l}\text { Diferentes niveles de ansiedad en función del } \\
\text { instrumento del músico } \\
\text { El tratamiento con medicamento es más barato que } \\
\text { la intervención psicológica (CBT) } \\
\text { A largo plazo la CBT es más eficaz y no tiene } \\
\text { efectos secundarios. }\end{array}$ \\
\hline
\end{tabular}




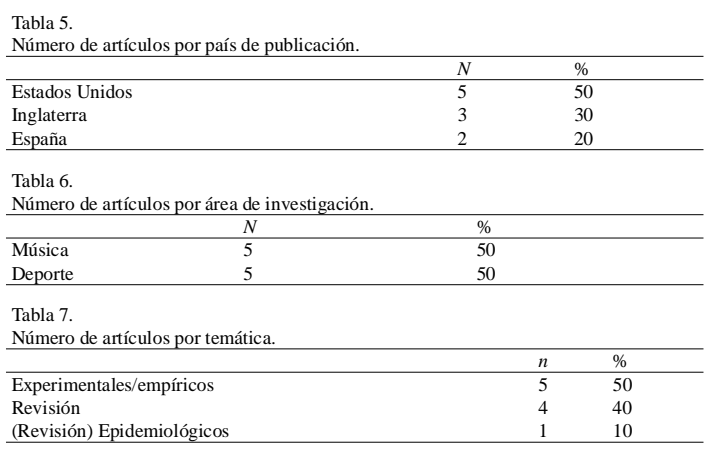

En cuanto al área de investigación, podemos observar en la Tabla 6 aproximadamente aquí, cómo de los artículos seleccionados, el 50\% hace referencia a la ansiedad en los músicos y el $50 \%$ a la ansiedad en los deportistas.

En la Tabla 7 podemos observar cómo el 50\% de los artículos están relacionados con formas de tratamiento y/o intervención de la ansiedad que sufren tanto deportistas como músicos. Únicamente el $40 \%$ de los artículos intentan explicar la relación entre el rendimiento y la ansiedad y proponer modelos explicativos. El 10\% restante procede de estudios puramente epidemiológicos sobre la ansiedad por el rendimiento.

\section{Discusión}

Después de la búsqueda, selección y lectura de los artículos que se ha llevado a cabo con una revisión sistemática de la literatura, podemos ver claramente, de que a pesar de ser un tema candente en la psicología y en las ciencias del rendimiento, no existen suficientes estudios al respecto con garantías científicas y que se hayan publicado en revistas presentes en la base de datos que controla el impacto científico de los mismos. Asimismo, podemos observar como los estudios se dirigen a dos campos distintos, pero con ciertas similitudes (Martens, 1990) como son la música y el deporte. Cabe recalcar que nuestro objetivo principal ha sido el ámbito deportivo, pero que hemos abierto la posibilidad de analizar también los del ámbito musical por las similitudes entre ambos.

En nuestra búsqueda por esclarecer las relaciones existentes entre la ansiedad y el rendimiento deportivo, nos topamos con visiones diferentes sobre cómo son dichas relaciones. Por un lado, se abre la posibilidad de que la ansiedad cómo tal no sea siempre un aspecto debilitador del rendimiento, sino que por el contrario bajo determinadas condiciones pueda, incluso favorecer dicho rendimiento.

Por otro lado, también se abre la posibilidad de que la ansiedad, en sus formas, cognitiva, somática, y motora, no afecte de la misma manera al rendimiento.

En el trabajo de Raglin y Turner, de 1993, dichos autores realizaron un estudio descriptivo, para comparar los dos modelos teóricos predominantes que relacionaban ansiedad y rendimiento. Estos modelos (la teoría de la U-invertida, con sus respectivas revisiones, y la Zona de Funcionamiento Óptimo), sostienen premisas diferentes, puesto que el primero alega que puntuaciones altas o bajas en nivel de activación/ ansiedad pueden repercutir negativamente en el rendimiento, y por otro lado puntuaciones moderadas, aumentan el rendimiento.

En el trabajo de Raglin y Turner, se usan dos de las revisiones, una basada en la especificidad de la tarea, y otra en la individualidad del sujeto.

Por el contrario, la ZOF, sostiene que cada individuo necesita un nivel de activación/ansiedad específica, y que por tanto no existen puntuaciones «normativas».

Los resultados del estudio apoyan la teoría de la Zona de Funcionamiento Óptimo, por delante de la teoría de la U-invertida en cualquiera de sus variantes, puesto que las ZOF de los sujetos corresponden con las áreas de ansiedad alta/baja de la U-invertida, lo cual no concuerda con el supuesto de la necesidad de tener una ansiedad moderada para rendir almáximo.
En un estudio posterior (Turner \& Raglin, 1996), donde se examinaba la variabilidad de la ansiedad pre-competitiva y su relación con el rendimiento deportivo en corredores de atletismo, quisieron poner a prueba los dos modelos teóricos predominantes, y citados anteriormente.

Los resultados pusieron de manifiesto que los sujetos que tenían valores de ansiedad pre-competitiva, que coincidían con su ZOF obtenían mejor rendimiento deportivo. También se puso de manifiesto que existe gran variabilidad de ZOF entre sujetos. El deterioro del rendimiento se daba de igual forma cuando las puntuaciones de ansiedad precompetitiva estaban tanto por encima como por debajo de la ZOF, aunque la causa de este suceso no pudo ser determinada por el estudio. Por tanto, dicho trabajo da soporte a la ZOF, y no se encontraron evidencias que apoyen a la teoría de la U-invertida.

En otro estudio (Thelwell \& Maynard, 1998) con jugadores de cricket pusieron también a prueba la relación entre ansiedad y rendimiento, comparando los dos modelos teóricos mencionados antes, pero añadiendo al tipo de ansiedad (cognitiva o somática) la dirección de esta (favorecedora o debilitadora del rendimiento). Los resultados pusieron de manifiesto que puntuaciones por encima de la ZOF y por debajo de la misma, obtenían mejores resultados de rendimiento que aquellos que puntuaron «dentro» de la zona. Por lo tanto, este trabajo no da soporte a la ZOF.

El último estudio de revisión hasta la fecha (Patel, Omar \& Terry, 2010), se centra en la descripción, evaluación e intervención de la ansiedad por el rendimiento en atletas femeninas jóvenes, se pone de manifiesto que la ansiedad por el rendimiento es algo común y especialmente en las chicas, y más si estas son jóvenes, y que la severidad y persistencia de los síntomas está influida por diferentes factores. Propone la intervención multimodal integrando el tratamiento psicológico, y la medicación siempre que sea considerada necesaria por el médico.

En otro estudio que se centra en la intervención cognitiva basada en pensamientos positivos, en jugadores de fútbol semi-profesional, sobre la ansiedad y el rendimiento (Maynard \& Smith, 1993), se pone de manifiesto que la ansiedad cognitiva y somática están relacionadas, y que en la línea de los trabajos de Borkovec (1976), un cambio en uno de los dos componentes, provoca un cambio en el otro. Lo que no ha podido demostrar este estudio ha sido que reduciendo la ansiedad haya una mejoría en el rendimiento. Proponen que la ausencia de esta relación entre ansiedad y rendimiento puede deberse a que no sea un componente predictor del rendimiento en un deporte de equipo como el fútbol.

Después de esto, y atendiendo a las similitudes que existen entre rendimiento deportivo y musical, nos adentramos en el análisis de los artículos que hacen referencia a este último.

El campo del rendimiento musical, comparte ciertas similitudes con el rendimiento deportivo tal como se mencionó anteriormente. Por lo que nos ha parecido correcto, en la línea del trabajo de Martens, analizar también la literatura que hemos encontrado al respecto.

El primer artículo que nos encontramos data del 1991 y trata sobre la ansiedad por el rendimiento en músicos, fuente de importantes consecuencias negativas, que debe ser reducida para el buen rendimiento de dichos músicos, aportando metodologías de intervención psicológicas basadas en estrategias conductuales y/o cognitivas (Clark \& Agras, 1991).

El siguiente trabajo que encontramos en la revisión, data del 2005y también está enfocado al tratamiento de la ansiedad por el rendimiento, haciendo una revisión y análisis de los tratamientos disponibles hasta la fecha, concluyendo positivamente sobre la eficacia de la exposición y la reestructuración cognitiva como tratamientos de elección. (McGinnis \& Milling, 2005).

Los últimos estudios sobre ansiedad en el rendimiento en músicos, datan de 2011, dato, que parece indicar el creciente interés sobre este fenómeno en elámbito musical.

En el trabajo de Robert Allen (2011), se pone de manifiesto cómo la improvisación parece tener efectos positivos sobre la ansiedad por el rendimiento, y lo propone como una herramienta terapéutica más en el tratamiento para dicha ansiedad. Además, argumenta, que los estudian- 
tes de piano (muestra del estudio), padecen más ansiedad por el rendimiento al interpretar una pieza clásica (conocida por el público), que llevar a cabo una improvisación delante del mismo.

Los dos últimos estudios (Brugués, 2011a, 2011b) son una revisión sistemática de los trabajos sobre epidemiología y sobre opciones de tratamiento de la ansiedad por el rendimiento en músicos respectivamente. El primer trabajo pone de manifiesto, la alta tasa de prevalencia de la ansiedad por el rendimiento, sobre todo en etapas iniciales de la práctica musical y a edades tempranas. El segundo trabajo propone el tratamiento psicológico cognitivo conductual, por delante del tratamiento con medicación (propanolol), ya que, aunque el tratamiento médico es más barato, a largo plazo los resultados derivados de la intervención de la terapia cognitivo conductual, son más duraderos.

Parece pues relevanteresaltar la importancia que se le da al constructo ansiedad, en el campo de la psicología del rendimiento humano, ya sea en el área deportiva o en el área musical, cosa que se pone de manifiesto en el igual número de trabajos que hemos manejado en uno u otro ámbito.

Existen numerosos trabajos que exploran desde diferentes perspectivas las relaciones entre ansiedad y rendimiento, aunque en líneas generales, destaca la concepción, de que la elevada ansiedad influye negativamente en el rendimiento, ya que existen multitud de trabajos en los que se trata de intervenir, para reducir la ansiedad, de forma implícita o explícita.

Si bien es cierto que la ansiedad ejerce algún efecto sobre el rendimiento, no quedan del todo claras las relaciones que se establecen entre ambas variables, empezando por la posibilidad de que la ansiedad influya de manera diferente en función del individuo y de su percepción de la misma como facilitadora o debilitadora del rendimiento, y pasando por el tipo de deporte y sus características intrínsecas, si es deporte individual, o de equipo, requisitos físicos del mismo etc. (García-Mas et al. 2011).

Del conjunto de trabajos analizados, sólo tres de ellos, comparan modelos teóricos para explicar las relaciones entre ansiedad y rendimiento, y sus resultados, en líneas generales parecen decantarse a favor del modelo de la ZOF, por delante del modelo de la U-invertida, para el cual no se han encontrado evidencias. A pesar de ello, no son trabajos suficientes como para aceptar un modelo por delante del otro.

Otro dato a tener en cuenta, es que no existen en lengua española, trabajos que analicen dichas relaciones, cosa por otra parte entendible, si setiene en cuenta quela lengua vehicular de la ciencia es el inglés, pero no deja de ser un dato llamativo. En la línea de lo mencionado, es llamativo el hecho de que la mayoría de los trabajos de esta revisión (70\%) son de regiones anglosajonas, quizás por el peso que el rendimiento tiene en dichas culturas.

En cuanto al campo concreto de la música, llama la atención el creciente interés por la ansiedad por el rendimiento en músicos, en la última década, ya que la mayoría de trabajos de la muestra, datan del 2005 en adelante. Hay que destacar, sin embargo, que la mayoría de trabajos se centran en la intervención y tratamiento de la ansiedad para su reducción, y no existen estudios sobre modelos teóricos (ZOF o Uinvertida, por ejemplo), que intenten explicar la relación entre variables.

En líneas generales, parece pues que, la relación entre ansiedad y rendimiento, interesa, y que muchos de los trabajos se centran en revertir una situación problemática (una vez que la ansiedad ha aparecido), y faltan trabajos sobre la prevención de dicha ansiedad además de que son pocos los trabajos que contrastan modelos teóricos.

Sin embargo, otra problemática, que se deduce de los trabajos citados, es referente al concepto de rendimiento, ya que este, lejos de ser un constructo unitario y global se muestra como un concepto inconsistente a lo largo de todos los artículos analizados.

Si nos centramos en rendimiento objetivo, nos referimos a aquellas medidas cómo tiempos, mejores marcas, distancias recorridas, número de pases acertados, puntos logrados, goles marcados etc.

Por otro lado, existe también la posibilidad de analizar el rendimiento desde una perspectiva subjetiva, vista como el bienestar percibido por el deportista durante la ejecución de la tarea, aunque, por último, se puede utilizar un enfoque mixto, que tenga en cuenta y combine ambos tipos de medidas (tanto objetivas como subjetivas).

Además, y dado que el rendimiento deportivo alude al resultado en un deporte dado, y que engloba las esferas física, técnica, táctica, y psicológica, no podemos sino, aceptar que el concepto de rendimiento, debe adaptarse a cada actividad concreta con sus singularidades.

Para futuros trabajos, se debería seguir poniendo a prueba los dos modelos teóricos predominantes, teniendo en cuenta las diferencias entre deportes, ya que no parecen suficientes los trabajos publicados sobre las relaciones entre ansiedad y rendimiento cómo para dar por aceptar uno y otro modelo teórico.

En relación al concepto de rendimiento, se debería profundizar más en la definición del mismo, para hacerlo un constructo más consistente y específico.

Además, habría que profundizar en si el deporte que se practica, guarda relación con la ansiedad experimentada, y en cómo esta repercute en el rendimiento en dicho deporte, ya que quizás en determinados deportes afecte más un tipo que otro, de manifestación ansiógena, ya sea esta una respuesta de tipo una respuesta de tipo fisiológica, motora o cognitiva.

Siguiendo el mismo esquema mencionado, en el ámbito de la música, todo y el creciente interés por la relación entre la ansiedad y el rendimiento musical de los últimos años (tenemos igual número de artículos deportivos que musicales), no parece existir ninguna base empírica que intente poner a prueba los modelos teóricos existentes en dicho campo de trabajo.

De la misma forma, que en el terreno deportivo es de menester analizar las relaciones entre ansiedad y rendimiento en función de la tarea y del deporte concreto, sería pues, de igual forma interesante, profundizar en los diferentes «actores» musicales, en función del instrumento o de la familia de instrumentos que toca cada uno.

Es pues necesaria una revisión de los modelos teóricos que explican las relaciones entre ansiedad y rendimiento y una revisión profunda del concepto de rendimiento.

\section{Conclusiones}

Nos encontramos pues, que la relación entre ansiedad y rendimiento no está del todo clara, con dos marcos teóricos predominantes en los artículos (IZOF y U-Invertida), de los cuales no existen suficientes estudios, así como tampoco están claramente establecidas las relaciones que se establecen entre la ansiedad y el rendimiento, y, por último, si verdaderamente la ansiedad influye o no sobre este último (y en qué sentido) (León-Prados, Fuentes \& Calvo, 2014).

Es importante destacar, la fragilidad y poca consistencia del término performance, puesto que abarca una gran variedad de comportamientos. A veces cuando se habla de rendimiento se hace aludiendo a los resultados conseguidos, otras sin embargo se hace teniendo en cuenta los procesos realizados, y en otra se alude al bienestar del atleta durante la competición, lo cual provoca que sea difícil establecer ningún tipo de relación de dicha variable con cualquier otra por la ambigüedad de la misma. En otros casos, se puede aludir al rendimiento haciendo alusión al término habilidad motriz como en el estudio con sujetos en edad escolar que relacionaba este último con la ansiedad experimentada antes y después de una práctica deportiva de escalada (Pérez, Hernández \& García, 2011), y en otras a veces se diferencia entre el rendimiento auto y hetero percibido en función de quién evalúa dicho rendimiento (Bohórquez, Delgado \& Fernández, 2017).

Si bien queda claro también, por el volumen de trabajos realizados al respecto, que a nivel «popular» la ansiedad es un problema que afecta al rendimiento, dada la gran cantidad de trabajos enfocados a lidiar con dicho problema.

Además, también es destacable es que la mayoría de trabajos provienen de la cultura anglosajona, debido, quizás, a la importancia que otorgan al rendimiento en diferentes ámbitos, así como en el origen editorial de las revistas que se hallan indexadas y con Factor de Impacto enlaWoS. 
Parece pues evidente que además de redefinir el término performance, para aportar un concepto más concreto y aceptado globalmente, quizás haya llegado el momento de superar los modelos explicativos tradicionales, ya que parecen no poder explicar la relación compleja que existe entre el rendimiento y la ansiedad, en favor de nuevos modelos explicativos que tengan en cuenta variables de tipo individual y deportivo, y su relación con otras variables.

El presente trabajo, no está exento de limitaciones. La primera de ellas, es la imposibilidad de no haber podido llevar a cabo un metaanálisis debido a la gran dispersión de datos que encontramos y la diversidad metodológica que existe. Esto no ha permitido extraer conclusiones estadísticas más sólidas.

Además, el hecho de haber seleccionado una única base de datos, puede haber producido un sesgo en las conclusiones, puesto que es más que probable que, a pesar de que WoS es una de las bases de datos con mayor número de publicaciones, hayamos pasado por alto alguna que fuera relevante, y que esté alojada en otra base de datos como SCOPUS, por ejemplo. En este sentido, sería interesante complementar estos dos análisis con otro llevado a cabo sobre la base Journal Scholar Metrics, para incluir las revistas españolas en el mismo.

\section{Referencias}

Botella, J. \& Gambara, H. (2012). Qué es el meta-análisis, (2a. edición) Madrid: Biblioteca Nueva.

Buceta, J. (2001). Psicología del entrenamiento Deportivo, (3ra. edición) Madrid: Dykinson

Bohórquez, M., Delgado, P. \& Fernández, J. (2017). Rendimientos deportivos auto y hetero percibidos y cohesión grupal: un estudio exploratorio. Retos, 31, 103-106.

Borkovec, T. (1976). Physiological and cognitive processes in the regulation of anxiety. Consciousness and self-regulation:Advances in research, 261-312.

Fechner, G (1966). Elements of Psychophysics. Nueva York: Holt, Rinehart \& Winston.

García-Mas, A., Palou, P., Smith, R., Ponseti, X., Almeida, P., Lameiras, J., Jiménez, R. \& Leiva, A. (2011). Ansiedad competitiva y clima motivacional en jóvenes futbolistas de competición, en relación con las habilidades y el rendimiento percibido por sus entrenadores. Revista de Psicología del Deporte, 20 (1), 197-207.

García-Mas, A., Rubio, V., Fuster-Parra, P., Pujals, C. \& Núñez, A. (2014). Determinación de las variables psicológicas y deportivas relevantes a las lesiones deportivas: Un análisis bayesiano. Revista de Psicología del Deporte, 23(2), 423-429.

Glass, G.V. (1976). Primary, secondary, and metaanalysis of research. Educational Researcher, 10, 3-8.

Gutiérrez, M., Estévez, A., García, J. \& Pérez, H. (1997). Ansiedad y rendimiento atlético en condición de estrés: efectos moduladores de la práctica. Revista de Psicología del Deporte, 6(2), 27-46.

Hanin, Y.L. (1978). A study of anxiety in sports. Sport psychology: An analysis of athletic behavior, (pp. 236-249). Ithaca, NY: Straub

León-Prados, J.A.; Fuentes, I. y Calvo, A. (2014). Relación entre ansiedad estado, autoconfianza percibida y rendimiento en baloncesto. Revista Internacional de Medicina y Ciencias de la Actividad Física y el Deporte, 14(55), 527-543.

LeUnes, A., Hayward, S.A., \& Daiss, S. (1988). Annotated bibliography on the Profile of Mood States in sports. Journal of Sport Behavior, $11,213-240$.

Martin, D. (2001). Manual de metodología del Entrenamiento Deportivo. Barcelona: Paidotribo.

Martens, R. (1990). Competitive Anxiety in Sport. Champaign, IL: Human Kinetics.

Oxendine, J.B. (1970). Emotional arousal and motor performance. Quest, 13, 23-32.

Pérez, R., Hernández, E. \& García, I. (2011). Relación entre el nivel de habilidad motriz y la ansiedad percibida antes y después de una práctica des escalada en niños de Educación Primaria. Retos. Nuevas tendencias en Educación Física, Deporte y Recreación, 19, 25
29.

Ponseti, F.J., García-Mas, A., Cantallops, J. \& Vidal, J. (2017). Diferencias de sexo respecto de la ansiedad asociada a la competición deportiva. Retos, 31, 193-196.

Ponseti, F.J., Sesé, A. \& García-Mas, A. (2016). The impact of competitive anxiety and parental influence on the performance of young swimmers. Revista Iberoamericana de Psicología del Ejercicio y el Deporte, 11(2), 229-237.

Pozo, A., Cortes, B. \& Martín, A. (2013). Conductancia de la piel en deportes de precisión y deportes de equipo. Estudio preliminar. Revista de Psicología del Deporte. 22(1), 19-28.

Ramis, Y., Torregrosa, M., Viladrich, C. \& Cruz, J. (2010). Adaptación y validación de la versión española de la Escala deAnsiedad Competitiva SAS-2 para deportistas de iniciación. Psicothema. 22(4), 1004-1009.

Ruiz-Juan, F., Zarauz, A., Flores-Allende, G. (2016). Ansiedad precompetitiva en corredores de fondo en ruta en función de sus variables de entrenamiento. Retos, 30,110-113.

Sánchez, A., González, E. Ruiz, M., San Juan, M., Abando, J., De Nicolas, L. \& García, F. (2001). Estados de ánimo y rendimiento deportivo en fútbol. ¿Existe la ventaja de jugar en casa? Revista de Psicología del Deporte.10 (2), 197-209.

Smith, R.E., \& Smoll, F.L. (2004). Anxiety and coping in sport: Theoretical models and approaches to anxiety reduction. En T. Morris \& J.J. Summers (Eds.), Sport psychology: Theories, applications, and issues (2), (pp. 294-321) Sydney,Australia: Wiley.

Sonstroem, R.J. \& Bernardo, P. (1982). Intraindividual pregame state anxiety and basketball performance: Are-examination of theinvertedU curve. Journal of Sport Psychology, 4, 235-245.

Yerkes, R. \& Dodson, J. (1908). The relation of Strength of Stimulus to Rapiity of Habit-Formation. Journal of Comparative Neurology and Psychology, 18, 459-482.

Wundt, W. (1874). Grundzüge der physiologischen Psychologie. Leipzig: Engelmann.

Artículos que han formado parte de la revisión sistemática

Allen, R. (2011). Free improvisation and performance anxiety among piano students. Psychology of Music, 41(1), 75-88.

Brugues, A. (2011). Music Performance Anxiety- Part 1. A Review of its Epidemiology. Medical Problems of Performing Artists, 26(2), pp. 102-105

Brugues, A. (2011). Music Performance Anxiety- Part 2: A Review of Treatment Options. Medical Problems of Performance Artists. 26(3), 164-171

Clarck, D., \& Agras, W. (1991). The assessment and treatment of performance anxiety in musicians. American Journal of Psychiatry. 148(5), 598-605.

Maynard, I., Warwickevans, L., \& Smith, M. (1995). The effects of a cognitive interventions strategy on competitive state anxiety and performance in semiprofessional soccer players. Journal of Sport \& Exercise psychology. 17, 428-446.

McGinnis, A. \& Milling, L. (2005).Psychological treatment of musical performance anxiety: Current status and future directions. Psychotherapy. 42(3), 357-373.

Patel, D., Omar, H., \& Terry, M. (2010). Sport-related Performance Anxiety in Young Female Athletes. Journal of Pediatric and adolescent gynecology. 23, 325-335.

Raglin, J., \& Turner, P. (1993). Anxiety and performance in track and field athletes- A comparison of the inverted-U hypothesis with zone of optimal function- theory. Personality and Individual Differences. 14(1), 163-171.

Turner, P., \& Raglin, J. (1996). Variability in precompetition anxiety and performance in college track and field athletes. Medicine \& Science in Sports \& Exercise. 28(3), 378-385.

Thelwell, R., \& Maynard, I. (1998). Anxiety-performance relationships in cricketers: Testing the zone of optimal functioning hypothesis. Perceptual and Motor Skills. 87, 675-689. 